\title{
Modulating Electronic Structures of Armchair GaN Nanoribbons by Chemical Functionalization under an Electric Field Effect
}

\author{
Naresh Alaal and Iman S. Roqan*(-) \\ Physical Sciences and Engineering Division, King Abdullah University of Science and Technology, Thuwal 23955-6900, Saudi Arabia
}

Supporting Information

ABSTRACT: The electronic and magnetic properties of oxygen- and sulfur-passivated one-dimensional armchair GaN nanoribbons (A-GaNNRs) are revealed using both firstprinciples density-functional theory and $a b$ initio molecular dynamics simulations. We explore that an applied external electric field can further modulate the electronic properties of both pristine and passivated A-GaNNRs, thus changing their properties (semiconducting-metallic-half-metallic). A-GaNNRs of $0.9-3.1 \mathrm{~nm}$ width are subjected to further investigations, which reveal that sulfur termination transforms pristine A-GaNNRs from direct into indirect band gap semiconductors, without affecting their nonmagnetic nature. On the other hand, oxygen passivation introduces spin-polarized behavior with a finite magnetic moment. Magnetism characteristics in both bare and sulfur-passivated A-

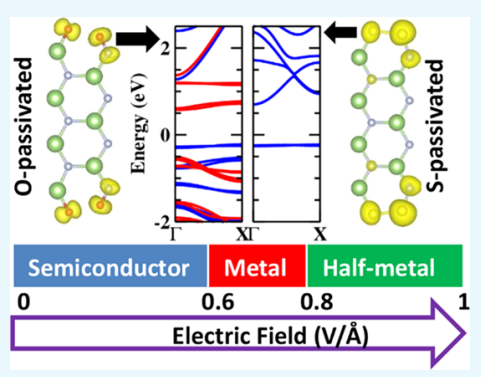
GaNNRs are induced by applying a critical electric field along the direction of NR width. The passivated A-GaNNRs are more stable compared to bare ones, while sulfur-passivated A-GaNNRs exhibit higher stability at higher temperatures $\left(>500{ }^{\circ} \mathrm{C}\right)$. Thus, our results suggest that A-GaNNRs can be used in a broad range of electronic, optoelectronic, and spintronic applications.

\section{INTRODUCTION}

The discovery of graphene ${ }^{1}$ and its unique properties ${ }^{2-4}$ prompted extensive research into two-dimensional (2D) materials, as they possess distinct physical characteristics compared with their three-dimensional (3D) counterparts. 2D monolayers (MLs) such as transition-metal dichalcogenides, phosphorene, borophene, silicene, and Mxenes have been synthesized successfully. ${ }^{5-10}$ On the other hand, onedimensional (1D) nanostructures garnered significant attention from researchers due to their potential optoelectronic, photochemical, and electro-transport properties caused by quantum confinement and reduced dimensionality. ${ }^{11-16}$ Particularly, 1D nanoribbons (NRs) generated from 2D materials display intriguing electronic properties based on their width and edge configurations and are, thus, considered to be potential building blocks for the next generation of electronic devices. ${ }^{17-22}$ Based on the crystal orientation, NRs are typically explored in armchair or zigzag edge configurations. $^{23}$

It is well known that III-nitrides are wide-band-gap semiconductors and have the potential for use in optoelectronic and high-power applications, such as lasers, lightemitting diodes, and detectors, as well as devices operating in harsh environments. ${ }^{24-28}$ Recent developments have shown that 3D GaN can form a hexagonal 2D graphene-like structure with $\mathrm{sp}^{2}$ hybridization with an indirect band gap structure. ${ }^{29,30}$ Buckled 2D GaN has been synthesized using a grapheneencapsulation technique. ${ }^{31}$ Single-crystalline $1 D$ GaNNRs (GaNNRs), which have thicknesses of a few nanometers, have been synthesized successfully by flowing ammonia on $\mathrm{Ga}_{2} \mathrm{O}_{3}$ thin films. ${ }^{32}$ Some recent theoretical investigations pertaining to bare-edge and hydrogen-passivated armchair
GaNNRs (A-GaNNRs) suggested that both NRs are nonmagnetic (NM) direct band gap semiconductors, whereas bare-edge zigzag GaNNRs (z-GaNNRs) are metals irrespective of their width. ${ }^{33}$ Chemical functionalization of $\mathrm{z}$-GaNNR edges with hydrogen and fluorine transforms them into semiconducting and half-metallic materials, respectively. ${ }^{34}$ Moreover, investigations into doping effects have shown that a carbon dopant is preferentially substituted at edge $\mathrm{Ga}$ or $\mathrm{N}$ sites in A-GaNNRs and z-GaNNRs, respectively. ${ }^{35}$ However, most extant studies in this domain have focused on z-GaNNRs, while reports on the properties of A-GaNNRs are scarce. Furthermore, the effect of electric field on bare and chemically functionalized A-GaNNRs by different atoms (other than hydrogen or fluorine), as well as their thermal stability, has never been investigated, even though this is crucial for producing novel functional devices. Thus, there is still a need for exploring potential optical, electronic, and magnetic characteristics of A-GaNNR-based devices.

In this study, we address the aforementioned gaps in pertinent research by exploring the electronic and magnetic properties of A-GaNNRs by adopting for the first time edge oxidization and sulfurization, as well as applying an external field along the direction of the ribbon width. Our findings indicate that edge oxidization transforms the A-GaNNRs into magnetic semiconductors with a finite magnetic moment (MM), whereas sulfur passivation transforms them from direct into indirect band gap semiconductors. Moreover, application of an external electric field modulates the electronic structure

Received: November 12, 2019

Accepted: December 24, 2019 
of both bare and passivated A-GaNNRs. Ab initio molecular dynamics simulations (AIMD) reveal their structure stability at different temperatures.

\section{COMPUTATIONAL DETAILS}

All first-principles calculations reported in this work were performed using the Vienna Ab initio Simulation Package ${ }^{36,37}$ based on density-functional theory (DFT). The PerdewBurke-Ernzerhof $(\mathrm{PBE})^{38}$ functional coupled with the generalized gradient approximation was used for the exchange-correlation potentials. The electron-ion interaction was described using the projector-augmented wave method with a plane wave cutoff energy of $500 \mathrm{eV}$. All atoms comprising the unit cell or supercell were relaxed until the force on each atom decreased below $0.01 \mathrm{eV} / \AA ̊$. The energy tolerance between two consecutive electronic relaxation steps was considered to be $10^{-6} \mathrm{eV}$. A Monkhorst $k$-point scheme with $9 \times 1 \times 1$ and $25 \times 1 \times 1$ meshes was used for the structural relaxation and electronic structure calculations, respectively. The NRs were presented in the $x y$-plane with infinite periodicity in the $x$-direction. A vacuum layer of $>12 \AA$ thickness was considered in the $y$ - and $z$-directions to avoid interactions between adjacent cells. The following annotation was adopted for the nanoribbon models: $N_{\mathrm{a}}-\mathrm{X}-\mathrm{A}-\mathrm{GaNNR}$, where $N_{\mathrm{a}}$ denotes the NR width (along the $y$-direction) and $\mathrm{X}$ indicates the passivating atom. In this study, we investigated the widths in the $6<N_{\mathrm{a}}<20$ range, corresponding to the NR widths (along the $y$-direction) ranging from 0.9 to $3.1 \mathrm{~nm}$ for all A-GaNNRs. The crystal structures of the NRs and charge density plots were obtained using the VESTA software package. $^{39}$ The atomic positions were also relaxed in the presence of an external electric field, and a sawtooth-like potential was used. To examine the temperature effects on NR stability, we carried out AIMD simulations using $8 \times 1 \times 1$ supercells (112 and 144 atoms for bare and O/S-A-GaNNRs, respectively), which were modeled based on the initial unit cell geometry configurations. The Nosé-Hoover thermostat ${ }^{40}$ scheme was adopted for temperature control. The simulation time was set to 5 ps with a step size of $0.5 \mathrm{fs}$.

\section{RESULTS AND DISCUSSION}

Atomic Structure and Zero Electric Field Band Structure of Bare A-GaNNRs. Before presenting the results obtained through A-GaNNR investigations, it is worth noting that our calculations of the structural and electronic properties of a $2 \mathrm{D} \mathrm{GaN}$ monolayer (ML) yields a $\mathrm{Ga}-\mathrm{N}$ bond length of $1.86 \AA$, a lattice constant of $3.25 \AA$, and a band gap of $1.94 \mathrm{eV}$, in line with previously reported findings. ${ }^{29,41}$ An A-GaNNR model is constructed by cutting such a GaN ML in a particular direction. We explore the electronic properties of bare AGaNNRs in the absence of an external electric field, which are used for comparison, as will be discussed later. Moreover, these results also help benchmark the methodology adopted here against previously reported findings related to bare A-GaNNRs of certain widths.

The atomic structure of a bare A-GaNNR is presented in Figure $1 \mathrm{a}$, in which two edge $\mathrm{Ga}(\mathrm{N})$ atoms are labeled $\mathrm{Ga}_{1}$ $\left(\mathrm{N}_{1}\right)$ and $\mathrm{Ga}_{2}\left(\mathrm{~N}_{2}\right)$. Here, we present the results related to bare NRs (not doped, not passivated) of $N_{\mathrm{a}}=7$ (7-A-GaNNR) as an example, as we found that the NRs of different widths possess similar band structures and electronic properties (NR properties as a function of width will be discussed later). After

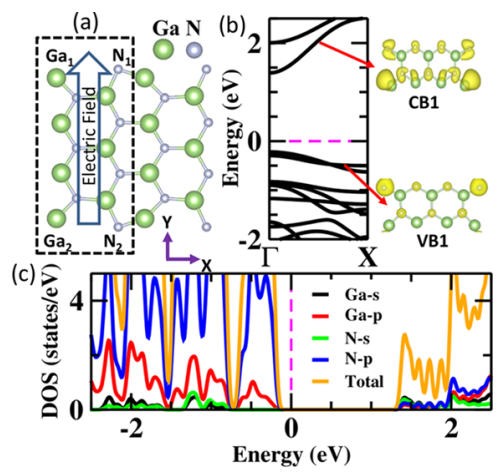

Figure 1. (a) Geometric structure, (b) band structure, and (c) projected and total density of states (DOS) of pristine 7-A-GaNNR. $\Gamma(0,0,0)$ and $X(0.5,0,0)$ are highly symmetrical Brillouin zone points. The partial charge densities of VB1 and CB1 of 7-A-GaNNR are presented in the right panel of (b). The dashed magenta line denotes the Fermi level $\left(E_{\mathrm{F}}\right)$.

structural relaxation, the optimized bond lengths of $\mathrm{Ga}-\mathrm{N}$ at the NR edges and in the inner region of the NR are 1.77 and $1.86 \AA$, respectively. While the former value is $0.09 \AA$ smaller than that observed in the $2 \mathrm{D} \mathrm{GaN} \mathrm{ML}$, the latter one is identical to that of the $2 \mathrm{D} \mathrm{ML}{ }^{29}$ The short $\mathrm{Ga}-\mathrm{N}$ bond length at the NR edges can be attributed to edge reconstruction, as the quantum dimension changes due to differences in applied strain or unpassivated dangling bonds. ${ }^{33}$ A similar feature has been observed in bare NRs obtained from other materials. ${ }^{18}$

The calculated band structure of $7-\mathrm{A}-\mathrm{GaNNR}$ is presented in Figure $1 b$, indicating that the NR is a semiconductor with a direct band gap $\left(E_{\mathrm{g}}=1.64 \mathrm{eV}\right)$. The valence band maximum (VBM) and the conduction band minimum (CBM) coincide at the highly symmetrical first Brillouin zone point $\Gamma$. The obtained band gap value is in good agreement with that previously reported for $7-\mathrm{A}-\mathrm{GaNNR}(1.8 \mathrm{eV})$ based on the DFT-LDA approach. ${ }^{33}$ The small difference between our PBE calculation and that obtained in the aforementioned study is attributed to the use of different exchange-correlation functionals. To determine the atomic contributions of the entire top valence band (VB1) and the entire bottom conduction band (CB1) of the NR in the first Brillouin zone, band-decomposed charge densities are presented in the right panel of Figure $1 \mathrm{~b}$. It can be seen that the VB1 is formed by dominant contribution of edge $\mathrm{N}$ atoms, whereas the interior $\mathrm{N}$ atoms provide limited contribution, as shown in the bottom right panel of Figure $1 \mathrm{~b}$. This is expected, as the dangling bonds attached to the edge $\mathrm{N}$ atoms trap free carriers due to missing atoms. The orbital projected density of states (PDOS) is shown in Figure 1c, which confirms that the VBM comes from the p-orbitals of the $\mathrm{N}$ atoms due to the higher electronegativity of $\mathrm{N}$, attracting the shared bonded electrons more easily than Ga. ${ }^{42}$ Moreover, the N-p state has a lower energy than that of $\mathrm{Ga}$, resulting in major contribution to the bonding states of the VBM. On the other hand, states pertaining to edge $\mathrm{Ga}$ and all $\mathrm{N}$ atoms within the NR contribute to $\mathrm{CB} 1$, as shown in the top right panel of Figure 1b. Particularly, the CBM comes from the s-orbitals of $\mathrm{Ga}$ atoms and s- and p-orbitals of $\mathrm{N}$ atoms (as shown in Figure 1c). Thus, this electronic structure allows the electronic and magnetic properties of the NRs to be explored when the edges are functionalized by chemical elements and under the influence of an external electric field. 
Band Structures of Bare A-GaNNRs under an External Electric Field. In this section, we discuss the effects of an externally applied electric field $\left(\vec{E}_{\text {ext }}\right)$, ranging from 0.1 to $1 \mathrm{~V} /$ $\AA$, on the band structures of bare A-GaNNRs. For consistency with the previous discussion, the reported results pertain to NRs with $N_{\mathrm{a}}=7$. In these calculations, the electric field is applied along the direction of NR width, i.e., $y$-direction of the unit cell, which is perpendicular to the NR growth direction, as shown in Figure 1a. Our findings reveal that no significant effect on the NR is obtained by applying the field along the $z$ direction, as the carrier (both electrons and holes) wavefunctions are restricted in the $x y$-plane in $2 \mathrm{D}$ materials. This result is in line with the findings previously reported for armchair and zigzag $\mathrm{MoS}_{2}$ NRs. ${ }^{14,19}$ Moreover, a positive electric field direction is assumed from the edge $\mathrm{Ga}_{2}$ and $\mathrm{N}_{2}$ to the edge $\mathrm{Ga}_{1}$ and $\mathrm{N}_{1}$.

Figure $2 \mathrm{a}$ and Figure $2 \mathrm{~b}$ present the band structures of $\mathrm{A}$ GaNNRs under electric field strengths of 0.2 and $0.6 \mathrm{~V} / \AA$,

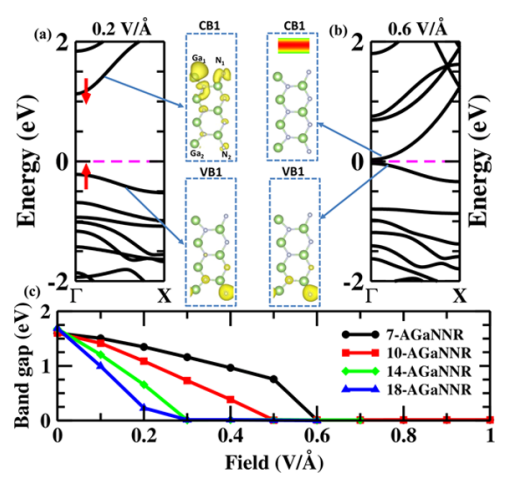

Figure 2. Band structures of 7-A-GaNNR for electric field strengths of (a) $0.2 \mathrm{~V} / \AA$ and (b) $0.6 \mathrm{~V} / \AA$. . The right panel of figure (a) and the left panel of figure (b) depict the partial charge densities of VB1 (bottom) and $\mathrm{CB} 1$ (top) at $0.2 \mathrm{~V} / \AA$ and $0.6 \mathrm{~V} / \AA$, respectively. The red arrows indicate that the $\mathrm{VB}$ moves up and the $\mathrm{CB}$ moves down due to field application, and the blue dashed box represents the unit cell of 7-AGaNNR. (c) Band gap as a function of external field strength for various $\mathrm{A}-\mathrm{GaNNR}$ widths.

respectively. When a weaker external electric field is applied, the NR band gap decreases compared to that under the zero electric field, as shown in Figure 2a. Increasing the field strength further decreases the band gap until it reaches the saturation point and vanishes at a critical electric field value $\left(\overrightarrow{E_{c}}\right)$, transforming the NR from a semiconductor into a metal, as shown in Figure 2b. Such a reduction in the band gap with an increase in electric field might be due to the quantumconfined Stark effect (QCSE). Under this effect, when the electric field is applied, the electron wavefunction in CB1 shifts against the electric field direction and the hole wavefunction in VB1 shifts along the electric field direction, decreasing the band gap value. A similar observation has been reported for carbon nanotubes and armchair boron nitride NRs (ABNNRs) and $\mathrm{MoS}_{2}$ NRs. ${ }^{14,43,44}$ Therefore, the difference in the electrostatic potential between the two edges created by the electric field may be attributed to the QCSE phenomena. According to the discussion in the previous section, the edge states play a key role in VB1 and CB1 formation in the AGaNNR band structure. In the band structure under the electric field of $0.2 \mathrm{~V} / \AA$ strength, VB1 is formed mainly from the edge $\mathrm{N}_{2}$ and the $\mathrm{N}$ atoms close to these $\mathrm{N}_{2}$, as shown in the bottom-right panel of Figure 2a. On the other hand, under the same field, CB1 is primarily derived from edge $\mathrm{Ga}_{1}$ and $\mathrm{N}_{1}$ atoms (top-right panel in Figure 2a), with a minor contribution from the remaining $\mathrm{N}$ atoms within the $\mathrm{NR}$. As the field strength is increased to $0.6 \mathrm{~V} / \AA$, the contribution of atoms to VB1 remains unchanged (bottom-left panel in Figure $2 \mathrm{~b}$ ). However, the states contributing to $\mathrm{CB} 1$ are located further away (the red/green band shown in the top-left panel of Figure 2b) and are weakly bound to the NR (representing significantly delocalized free carriers). These states lead to a rapid decrease in the band gap to zero, altering the semiconducting properties to metal-like characteristics, as shown in Figure 2b.

No significant change in the band gap is observed when the field polarity is reversed because atoms characterized by similar electronegativity are present at both edges. The band structure corresponding to the negative field strength $(-0.4 \mathrm{~V} / \AA)$ (in the sense defined earlier) shows the same band gap as that obtained for the corresponding positive field value (Figure S1 in the Supporting Information). The atomic contributions to VB1 and $\mathrm{CB} 1$ formation in the presence of a negative field remain the same as those under the influence of a positive field (Figure S1 in the Supporting Information).

To examine the phenomenon of material transformation from semiconducting into metallic-like nature for other NR widths, we also performed calculations for NRs of greater widths, as shown in Figure 2c. It can be seen that the band gap reduces as the electric field strength increases for all widths considered in this study and eventually vanishes under a strength of $\vec{E}_{\mathcal{c}}$, akin to the behavior observed for the $N_{\mathrm{a}}=7$ width. Indeed, as shown in Figure $2 c$, as the width increases, the value of $\left|E_{\mathrm{c}}\right|$ decreases. For example, the $\left|E_{\mathrm{c}}\right|$ value is $0.6 \mathrm{~V} /$ $\AA$ for $N_{\mathrm{a}}=7$, whereas it decreases to $0.3 \mathrm{~V} / \AA$ for $N_{\mathrm{a}}=18$. This phenomenon occurs because a smaller NR width induces a larger separation between the conduction band and the valence band levels due to greater quantum confinement. ${ }^{45}$ Such band gap behavior satisfies the condition proposed by Sanvito et al., ${ }^{14}$ confirming that the $\left|E_{c}\right|$ value required for band gap closure is inversely proportional to the NR width (Figure 2c). Interestingly, we further observed that across the entire electric field range examined in this study, the band gap nature is direct at the $\Gamma$ point until it vanishes. This finding is in contrast to other materials (such as armchair BNNRs and $\mathrm{MoS}_{2} \mathrm{NRs}$ ), where the CBM was found to move slightly away from the $\Gamma$ point toward the zone boundary $(\mathrm{X})$ as the electric field increases, leading to indirect band gap behavior. ${ }^{14,43}$

Magnetic Properties of Bare A-GaNNRs Induced by an External Electric Field. The presence of high DOS around the Fermi level near VB1 or CB1 transforms any nonmagnetic phase into a ferromagnetic (FM) one. ${ }^{46-48}$ This phenomenon prompted us to further explore the magnetic properties of the A-GaNNR by performing spin-polarized calculations under different external electric field strengths. Under the external electric field, the band structure of the AGaNNR splits into spin-up and spin-down channels, as shown in Figure 3a (7-A-GaNNR under $0.6 \mathrm{~V} / \AA ̊$ field strength), leading to a finite MM. The A-GaNNR exhibits ferromagnetic properties only under applied fields of strength exceeding the I $E_{\mathrm{c}} \mathrm{l}$ values (when the transition from the semiconducting to metallic NR nature occurs), as shown in Figure 3c. Figure 3a shows that both spin channels are metallic, owing to significant DOS at the Fermi level. In this case, the polarized carriers located near the Fermi level are fully delocalized and can be hybridized by other states, due to which the entire material 


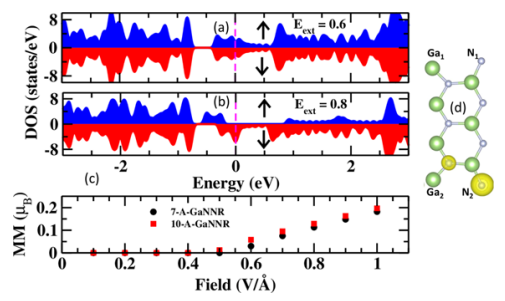

Figure 3. Spin-polarized density of states (DOS) of 7-A-GaNNR for field strengths of (a) $0.6 \mathrm{~V} / \AA$ and (b) $0.8 \mathrm{~V} / \AA$. The Fermi level has been set at $0 \mathrm{eV}$ and is represented by a dashed magenta line. (c) Magnetic moment (MM) of 7-A-GaNNR and 10-A-GaNNR as a function of field strength. (d) Spin density of a 7-A-GaNNR in the presence of a field of strength $0.8 \mathrm{~V} / \AA$.

matrix is polarized, inducing ferromagnetic properties. Interestingly, when the intensity of the electric field increases further, the material properties transform from metallic into half-metallic. In this case, one of the spin gaps (spin-up or spindown) exhibits semiconducting and the other metallic nature, $^{23}$ as shown in Figure $3 \mathrm{~b}$. Similar behavior was previously observed in zigzag graphene NRs and BNNRs. ${ }^{49-51}$ It is worth mentioning that the spin-polarized calculations are performed for the fields below the critical points and no significant change in the DOS is observed.

To determine the origin of MM, a spin density plot is drawn (Figure $3 \mathrm{~d}$ ), indicating that it is derived primarily from the edge $\mathrm{N}_{2}$, with a minor contribution from the $\mathrm{N}$ atom associated with the edge $\mathrm{Ga}_{2}$. The display of such diverse range of electronic properties modulated by the external electric field makes bare A-GaNNRs suitable for a wide range of applications. Such ferromagnetic behavior in these NRs would enable their use in spintronic device applications. Thus, modulating the electronic, structural, and magnetic properties by applying an external electric field allows obtaining bare AGaNNRs suitable for a wide range of applications.

Electronic and Magnetic Properties of $\mathrm{O}-$ and SPassivated A-GaNNRs. Under practical conditions, edge saturation is required to remove the dangling bonds in NRs as a means of enhancing their stability as well as removing defect states that trap carriers, resulting in enhanced electronic and optical properties. ${ }^{52}$ Available evidence indicates that edgefunctionalizing NRs with chemical elements (passivation) induces a wide range of electronic properties. ${ }^{53-55}$ For example, oxidization and sulfurization of graphene and phosphorene NRs have been investigated to enhance their properties. $^{56-58}$ In line with this prevalent trend, in this work, we present structural and electronic properties of A-GaNNRs passivated with oxygen and sulfur atoms. Figure $4 \mathrm{a}$ and Figure $4 \mathrm{~b}$ show the atomic structures of the passivated configurations, in which the passivating atoms $\mathrm{O}(\mathrm{S})$ attached to the edge $\mathrm{Ga}$ atoms are labeled $\mathrm{O}_{1}$ and $\mathrm{O}_{3}\left(\mathrm{~S}_{1}\right.$ and $\left.\mathrm{S}_{3}\right)$ and edge $\mathrm{N}$ atoms are denoted as $\mathrm{O}_{2}$ and $\mathrm{O}_{4}\left(\mathrm{~S}_{2}\right.$ and $\left.\mathrm{S}_{4}\right)$. For full correspondence with the discussions pertaining to the bare A-GaNNR, the results related to the O-A-GaNNRs and S-A-GaNNRs are presented for width $N_{\mathrm{a}}=7$. Figure $4 \mathrm{c}$ and Figure $4 \mathrm{~d}$ show the band structure associated with the configurations presented in Figure $4 \mathrm{a}, \mathrm{b}$, respectively, indicating that the band structure is modified through chemical passivation compared to bare AGaNNRs, which can lead to different electric, optical, and magnetic properties, as will be explained later.

We found that, after passivation, the $\mathrm{Ga}-\mathrm{N}$ bond length near the edges of $\mathrm{O}$ - and S-A-GaNNRs becomes 0.12 and 0.09

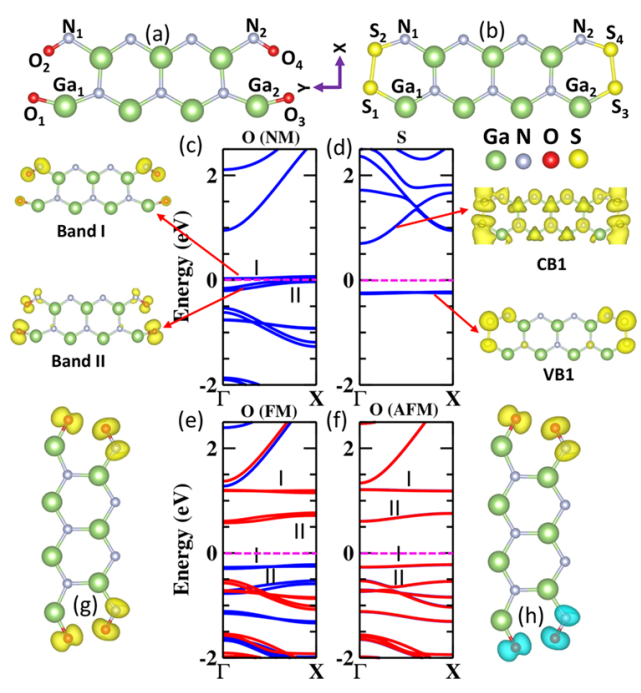

Figure 4. Geometric structures of (a) 7-O-A-GaNNR and (b) 7-S-AGaNNR. The band structures of (c) nonmagnetic (NM) 7-O-AGaNNR and (d) 7-S-A-GaNNR. The corresponding partial charge densities related to band types I and II are presented in the left panel of (c) and the partial charge densities of VB1 and CB1 of 7-S-AGaNNR are presented in the right panel of (d). The band structures of (e) ferromagnetic (FM) and (f) antiferromagnetic (AFM) 7-O-AGaNNR; the corresponding spin density plots of (g) FM and (h) AFM configurations of 7-O-A-GaNNR, respectively (yellow and blue colors represent spin-up and spin-down states, respectively).

$\AA$ greater than that obtained for the corresponding bare AGaNNRs, as shown in Table 1 . The $\mathrm{Ga}-\mathrm{O}, \mathrm{N}-\mathrm{O}, \mathrm{Ga}-\mathrm{S}$, and

Table 1. Bond Lengths of $\mathrm{Ga}-\mathrm{N}$ in the Inner Region (at the Edges) and Those Measured for $\mathrm{Ga}-\mathrm{S}, \mathrm{Ga}-\mathrm{O}, \mathrm{N}-\mathrm{S}$, and $\mathrm{N}-\mathrm{O}^{a}$

$\begin{array}{cccccccc}\text { structure } & \begin{array}{c}\mathrm{Ga}-\mathrm{N} \\ (\AA)\end{array} & \begin{array}{c}\mathrm{Ga}-\mathrm{S} \\ (\AA)\end{array} & \begin{array}{c}\mathrm{Ga}- \\ \mathrm{O} \\ (\AA)\end{array} & \begin{array}{c}\mathrm{N}-\mathrm{S} \\ (\AA)\end{array} & \begin{array}{c}\mathrm{N}-\mathrm{O} \\ (\AA)\end{array} & \begin{array}{c}E_{\mathrm{b}}(\mathrm{eV} / \\ \mathrm{X} \text { atom })\end{array} & \begin{array}{c}\delta \mathrm{G} \\ (\mathrm{eV} / \\ \text { atom })\end{array} \\ \begin{array}{c}\text { 7-A- } \\ \text { GaNNR }\end{array} & \begin{array}{c}1.86 \\ (1.80)\end{array} & & & & & & 0.37 \\ \begin{array}{c}\text { 7-O-A- } \\ \text { GaNNR }\end{array} & \begin{array}{c}1.86 \\ (1.92)\end{array} & & 1.74 & & 1.29 & -0.51 & 0.11 \\ \begin{array}{c}\text { 7-S-A- } \\ \text { GaNNR }\end{array} & \begin{array}{c}1.86 \\ (1.83)\end{array} & 2.19 & & 1.73 & & -0.71 & 0.13\end{array}$

${ }^{a}$ Binding energy $\left(E_{\mathrm{b}}\right)$ and Gibbs free energy of formation $(\delta G)$ of bare, O-, and S-A-GaNNRs for $N_{\mathrm{a}}=7$ (NR width).

$\mathrm{N}-\mathrm{S}$ bond lengths of O- and S-A-GaNNRs (1.74, 2.19, 1.64, and $1.28 \AA$, respectively) are presented in Table 1 . The short $\mathrm{N}-\mathrm{O}$ bond length confirms the presence of a double bond between the two atoms, and it matches the recently reported $\mathrm{N}-\mathrm{O}$ bond length in the O-doped 2D GaN ML. ${ }^{29}$ The distance $(2.27 \AA$ ) between two $\mathrm{O}$ atoms (see Figure $4 \mathrm{a}$ ) is wide enough to prevent bond formation between them, whereas a bond of 2.11 A length is achieved between $S$ atoms (see Figure $4 \mathrm{~b}$ ). Introduction of passivating atoms in the $\mathrm{O}$ and S-A-GaNNRs can make them exhibit different electronic properties compared to those of bare A-GaNNRs.

The binding energies of O-A-GaNNRs and S-A-GaNNRs were calculated using the following equation: ${ }^{59}$

$$
E_{\mathrm{b}}=\left(E_{\mathrm{X}-\mathrm{A}-\mathrm{GaNNR}}-E_{\mathrm{A}-\mathrm{GaNNR}}-n_{\mathrm{X}} * \mu_{\mathrm{X}}\right) / n_{\mathrm{X}}
$$

where $E_{\mathrm{X}-\mathrm{A}-\mathrm{GaNNR}}$ and $E_{\mathrm{A} \text {-GaNNR }}$ are the total energies of $\mathrm{X}$ passivated and bare NRs, respectively, $n_{\mathrm{X}}$ denotes the number 
of passivating atoms, and $\mu_{\mathrm{X}}$ is the chemical potential of the passivating atom $(\mathrm{X}=\mathrm{O}$ or $\mathrm{S})$. The negative binding energies indicate that both $\mathrm{O}$ and $\mathrm{S}$ atoms are strongly attached, preventing their disassociation from the edges. However, $S$ atoms are more strongly bound when compared with $\mathrm{O}$ atoms, as the binding energy of O-A-GaNNRs exceeds that characterizing S-A-GaNNRs (the obtained values are presented in Table 1).

To identify the magnetic phases that can be introduced in the material, spin-unpolarized and spin-polarized calculations are performed. The nonmagnetic (NM) band structure of $\mathrm{O}$ A-GaNNR is presented in Figure 4c, indicating metallic behavior, which is confirmed by the DOS shown in Figure S2 (Supporting Information), implying that $\mathrm{O}$ passivation can introduce extensive carrier states. Moreover, four bands can be noted around the Fermi level of the band structure of NM OA-GaNNR, two of which are degenerate (type I), and the remaining two are nondegenerate (type II), as shown in Figure $4 c$. The left panel of Figure $4 c$ shows the partial charge densities of the two types of bands, allowing us to investigate the metallic behavior caused by the bands at the Fermi level in the O-A-GaNNR in detail. As the unit cell of the NR is presented in the $x y$-plane, the $\mathrm{p}_{x}$ and $\mathrm{p}_{y}$ orbitals are located in the plane, whereas $\mathrm{p}_{z}$ orbitals are perpendicular to the plane. From the partial charge densities (left panel of Figure 4c), it is evident that type I bands are formed by nonbonding $\mathrm{p}_{z}$ orbitals of $\mathrm{O}_{1}$ and $\mathrm{O}_{3}$ and by hybridization of $\mathrm{O}_{2}-\mathrm{N}_{1}$ and $\mathrm{O}_{4}-\mathrm{N}_{2}$ bonds. These orbitals form flat bands at the Fermi level (Figure 4c). On the other hand, type II bands are formed by the $p_{x}$ orbital of all $\mathrm{O}$ atoms and edge $\mathrm{N}$ atoms. This finding is also supported by the PDOS of edge Ga and O atoms (Figure S2 in the Supporting Information).

Spin-polarized calculations show that the O-A-GANNR exhibits magnetic behavior. We considered both ferromagnetic (FM) and antiferromagnetic (AFM) configurations. The band structure presented in Figure $4 \mathrm{e}$ shows that the FM configuration of O-passivated NRs comprises different nondegenerate spin-up $(1.5 \mathrm{eV})$ and spin-down $(1.09 \mathrm{eV})$ band gaps. On the other hand, as indicated in Figure 4f, the AFM configuration displays only one band gap $(0.83 \mathrm{eV})$, as its spinup and spin-down bands overlap. The atomic contributions of type I and type II bands of FM and AFM phases are similar to those noted for the NM configuration, as shown in Figures S3 and $\mathrm{S} 4$ in the Supporting Information. The spin density plots of FM and AFM configurations of O-A-GaNNR are presented in Figure 4g,h, respectively, revealing that the $\mathrm{O}$ atoms provide the main magnetization contribution to both configurations, while the contribution from the edge $\mathrm{N}$ atoms attached to $\mathrm{O}$ is minimal. The FM configuration of O-A-GaNNR has a net MM of $3.12 \mu \mathrm{B}$ irrespective of width because, as mentioned above, only $\mathrm{O}$ and $\mathrm{N}$ atoms associated with the edges contribute to the MM. The $\mathrm{O}$ atoms attached to the edge $\mathrm{N}$ atoms have an $\mathrm{MM}$ of $0.68 \mu \mathrm{B}$, while those attached to the edge $\mathrm{Ga}$ atoms have an MM of $0.47 \mu \mathrm{B}$ at this width. On the other hand, the AFM configuration has a zero net MM because of the equal contribution from spin-up and spin-down states, as shown in Figure 4h. The MMs of $\mathrm{O}$ and $\mathrm{N}$ atoms $\left(\mathrm{O}_{2}\right.$ and $\left.\mathrm{N}_{1}\right)$ are antiferromagnetically aligned with the same atoms $\left(\mathrm{O}_{4}\right.$ and $\mathrm{N}_{2}$ ) at the opposite edge, as shown in the AFM spin density plot (denoted by blue and yellow), but their atomic MM values are equal to those obtained in the FM case.

The total energy difference $\Delta E$ between $\mathrm{FM}$ and $\mathrm{NM}$ configurations (FM-NM) of 7-O-A-GaNNR is thus $\Delta E=870$
meV per unit cell, favoring FM through $\mathrm{O}$ passivation. As this value is considerably higher than the energy at room temperature $(25 \mathrm{meV})$, it suggests that 7-O-A-GaNNR would retain strong magnetic features. For width $N_{\mathrm{a}}=7$, the FM phase energy is higher than that obtained for the AFM case $(\mathrm{FM}-\mathrm{AFM}=0.53 \mathrm{meV})$, and the difference becomes significantly smaller for larger widths (e.g., $0.04 \mathrm{meV}$ is obtained for $N_{\mathrm{a}}=20$ ). These results indicate that NRs of optimized width can be promising candidates for spintronic applications requiring strong and stable magnetic characteristics.

The S-A-GaNNR exhibits semiconductor characteristics in contrast to the O-A-GaNNR. However, the S-A-GaNNR has an indirect band gap nature $\left(E_{\mathrm{g}}=0.92 \mathrm{eV}\right)$, as its $\mathrm{CBM}$ and VBM occur at different highly symmetric Brillouin zone points, $\Gamma$ and X, respectively, as shown in Figure $4 d$. For the S-AGaNNR, the unpaired $p_{z}$ electrons of $S$ atoms at both edges $\left(S_{1}\right.$ and $S_{2}$, as well as those of $S_{3}$ and $S_{4}$ ) participate in bond hybridization. These $S$ bonds eliminate the bands formed by the orbitals of unbounded atoms at the edges. Consequently, S-A-GaNNRs exhibit nonmagnetic properties, as unpaired electrons are not available. These findings demonstrate that $S$ passivation transforms the band structure from a direct (in bare A-GaNNRs) into an indirect band gap without inducing any magnetic properties, as there are no states overlapping with the Fermi level, as shown in the PDOS of S-A-GaNNR (Figure S5 in the Supporting Information). VB1 originates from the edge passivating $\mathrm{S}$ atoms and edge $\mathrm{N}$ atoms and $\mathrm{CB} 1$ is derived from all atoms within the NRs apart from $\mathrm{Ga}$ atoms located at the NR edge, as shown in the right panel of Figure 4d.

Electric Field Effects on O- and S-Passivated AGaNNR Properties. The above discussion of the passivated NRs pertains to the case under zero electric field. Here, we discuss the combined effect of electric field and chemical passivation on the NRs. For O-passivated NRs, we consider only FM configurations to investigate the effect of the electric field on the O-A-GaNNR properties, as the energy difference between the FM and AFM configurations is very small. Similar to its bare counterpart, the band gap of the 7-O-A-GaNNR decreases as the field increases. Figure 5a presents the

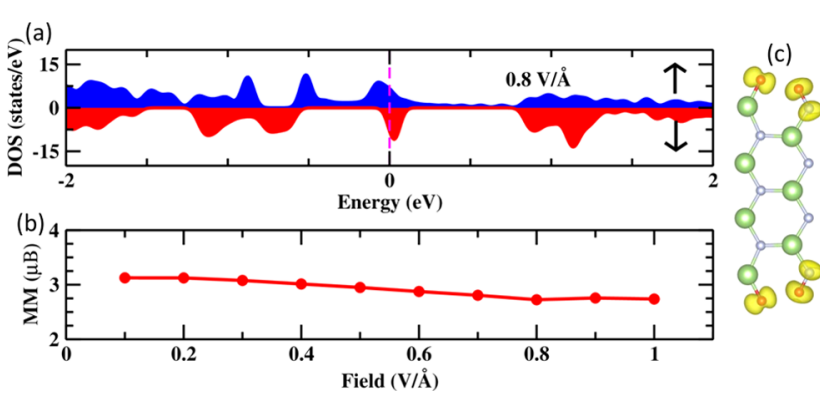

Figure 5. (a) DOS of 7-O-A-GaNNR for a field strength of $0.8 \mathrm{~V} / \AA$. (b) Magnetic moment (MM) of 7-O-A-GaNNR as a function of the external electric field. (c) The spin density plot of 7-O-A-GaNNR under a field strength of $0.4 \mathrm{~V} / \AA$.

electronic DOS of 7-O-A-GaNNR in response to a field strength of $0.8 \mathrm{~V} / \AA$, which shows that both spin-up and spindown channels exhibit metallic behavior. The DOS for lower field strengths $(0.1,0.3$, and $0.6 \mathrm{~V} / \AA)$ are shown in Figure S6 in the Supporting Information. The $\left|E_{\mathrm{c}}\right|$ value for this NR is $\sim 0.3 \mathrm{~V} / \AA$. As discussed in the preceding section, O-AGaNNRs are magnetic in the absence of the external field and 
retain this behavior when the electric field is applied. We also established that their magnetic contribution originates from the same atoms that contributed to the FM phase in the absence of the external field, as shown in Figure 5c. The MM of the NR decreases slightly when the electric field strength increases, as shown in Figure $5 b$.

For S-passivated NRs, the band structure of 7-S-A-GaNNRs under a field strength of $0.2 \mathrm{~V} / \AA$ is shown in Figure 6, where it

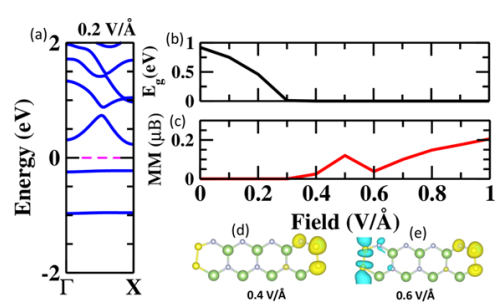

Figure 6. (a) Band structure of 7-S-A-GaNNR for a field strength of $0.2 \mathrm{~V} / \AA$. . (b) Band gap and (c) magnetic moment (MM) of 7-S-AGaNNR as a function of the external electric field. The spin density plots for 7-S-A-GaNNR at field strengths of (d) $0.4 \mathrm{~V} / \AA$ and (e) 0.6 $\mathrm{V} / \AA ̊$, respectively.

can be clearly seen that the band gap reduces gradually (Figure $6 \mathrm{~b})$, in line with the behavior observed in bare A-GaNNRs. We also found that the field strength $(0.3 \mathrm{~V} / \AA)$ required to transform a S-A-GaNNR into a metallic material (see Figure $S 7$ in the Supporting Information) is lower than that needed for its bare counterpart $(0.6 \mathrm{~V} / \AA)$, due to the smaller band gap in the passivated ones compared to bare ones.

Similar to the bare counterpart, the external electric field induces FM behavior in S-A-GaNNR when its strength exceeds the $\left|E_{\mathrm{c}}\right|$ value and the MM of S-A-GaNNR gradually increases as the field strength increases, as shown in Figure 6c. The NR MM reaches $0.12 \mu_{\mathrm{B}}$ for $0.5 \mathrm{~V} / \AA$, decreasing to $0.03 \mu_{\mathrm{B}}$ under $0.6 \mathrm{~V} / \AA$. The low MM corresponding to the field strength of $0.6 \mathrm{~V} / \AA$ is due to AFM spin alignment between the edges, as shown in Figure 6e. The net MM between the spin-up and spin-down states results in a lower magnetic moment value for this particular field strength. However, the MM maximum of $0.23 \mu_{\mathrm{B}}$ is obtained by further increasing the electric field to 1 $\mathrm{V} / \AA ̊$. The spin density plots presented in Figure $6 \mathrm{~d}$ show that one side of the edge $S$ atoms provides the main contribution to the MMs. Beyond the $\left|E_{c}\right|$ value, total energy differences suggest that the FM phase becomes more favorable than the $\mathrm{NM}$ phase as the field increases. For example, the $\Delta E$ values of 0.14 and $40 \mathrm{meV}$ are obtained when 0.5 and $1 \mathrm{~V} / \AA$ are applied to S-A-GaNNRs, respectively.

From the above discussion, it is evident that edge oxidization induces magnetic behavior in A-GaNNRs and modulates the band structure. On the other hand, edge functionalization with sulfur induces magnetic behavior in S-A-GaNNRs in the presence of an external electric field. Furthermore, bare AGaNNRs show half-metallic behavior when subjected to field strengths beyond the critical value. Thus, these findings indicate that A-GaNNRs can be utilized in the fabrication of a wide range of electronic, optoelectronic, and spintronic devices.

As the PBE functional underestimates the band gap values, we carried out calculations for several field strengths using the $\mathrm{HSE}^{60}$ functional, and the resulting band gap behavior is presented in Figure S8 in the Supporting Information. Thus, it is expected that, in practice, larger electric fields would be required to transform the material from a semiconductor into a metal compared to values yielded by the PBE calculations.

Width Dependence and Relative Thermal Stability of A-GaNNRs. Width Dependence. Thus far in this work, the band gaps of the bare and O- and S-passivated A-GaNNRs are presented only for the width of $N_{\mathrm{a}}=7$. Hence, the band gaps as a function of width in the absence of external electric field are discussed here. Figure 7 a presents the band gap nature for

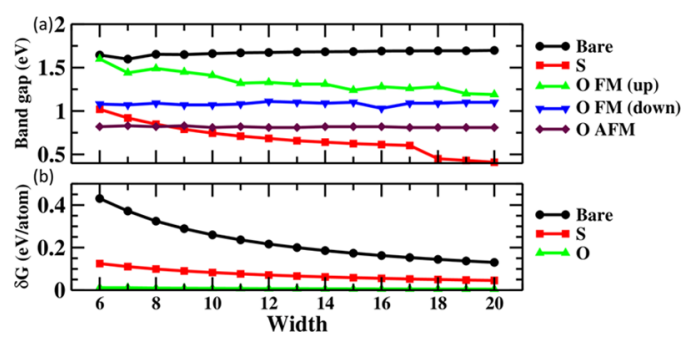

Figure 7. (a) Band gap values of the bare and O- and S-passivated AGaNNRs as a function of their width. The depicted spin-up and spindown band gaps pertain to the case of O-A-GaNNRs. (b) Formation energies of bare and $\mathrm{O}$ - and S-passivated A-GaNNRs as a function of the width.

the widths ranging from $N_{\mathrm{a}}=6$ to 20 for the three configurations, revealing that the resulting behavior coincides with that reported in the preceding sections. The band gaps of bare A-GaNNRs increase slightly as the NR width increases. On the other hand, the band gaps of S-A-GaNNRs decrease as the NR width increases. Moreover, for the FM phase of O-AGaNNRs, spin-up gaps increase and spin-down gaps decrease as a function of width, while the AFM phase band gaps are independent of the NR width.

Relative Thermal Stability. Thermal stability of the active material is one of the most important practical device characteristics. Consequently, we assessed the stability of both bare and passivated NRs by calculating the Gibbs free energies of NR formation $(\delta G)$ using the following equation: ${ }^{61}$

$$
\delta G=E_{\mathrm{c}}-x_{\mathrm{GaN}} \mu_{\mathrm{GaN}}-x_{\mathrm{O}} \mu_{\mathrm{O}} / x_{\mathrm{S}} \mu_{\mathrm{S}}
$$

where $E_{\mathrm{c}}$ is the cohesive energy per atom of the NR and $x_{\mathrm{GaN}}$, $x_{\mathrm{O}}$, and $x_{\mathrm{S}}$ are the molar fractions of the $\mathrm{GaN}$ dimer and $\mathrm{O}$ and $\mathrm{S}$ atoms, respectively; $\mu_{\mathrm{GaN}}$ denotes the chemical potential of the $\mathrm{GaN}$ dimer calculated by cohesive energy per unit cell of the $2 \mathrm{D} \mathrm{GaN} \mathrm{ML}$; and $\mu_{\mathrm{O}}$ and $\mu_{\mathrm{S}}$ represent the chemical potentials of the $\mathrm{O}$ and $\mathrm{S}$ atoms calculated from an isolated $\mathrm{O}_{2}$ molecule and $\mathrm{S}_{8}$ ring, respectively. The atomic structure of the $S_{8}$ ring was obtained from Materials Project. ${ }^{62}$

The calculated $\delta G$ values are presented in Table 1 . Generally, for any system, lower formation energy yields higher stability. The $\delta G$ values of the NRs in this study show that their stability can be increased by passivating the dangling bonds at the edges. For width $N_{\mathrm{a}}=7, \delta G$ obtained for the bare $\mathrm{NR}$ is 0.26 and $0.33 \mathrm{eV} /$ atom higher than the values obtained for the O- and S-A-GaNNR, respectively. Moreover, $\delta G$ for the $\mathrm{O}-\mathrm{A}-\mathrm{GaNNR}$ is $0.24 \mathrm{eV} /$ atom lower than that pertaining to the S-A-GaNNR, indicating that O-passivated NRs are the most stable among all NRs considered here. We also calculated the formation energies as a function of width, and the results presented in Figure $7 \mathrm{~b}$ indicate that the $\delta G$ of bare A-GaNNRs decreases with increasing width, suggesting that wider ribbons are more stable relative to the narrower ones. The $\delta G$ value of $\mathrm{S}-\mathrm{A}-\mathrm{GaNNR}$ decreases with increasing width but becomes 
independent of the NR width beyond $N_{\mathrm{a}}=12$. On the other hand, $\delta G$ of O-A-GaNNRs is weakly dependent on their width, but low formation energies indicate much higher stability, as shown in Figure $7 \mathrm{~b}$.

The formation energies $\delta G$ presented in the preceding section were calculated at zero temperature. To demonstrate the stability of A-GaNNRs at higher temperatures, we carried out AIMD stimulations at room temperature $(300 \mathrm{~K})$ and a high temperature $(800 \mathrm{~K})$, as shown in Figure 8 . Our findings

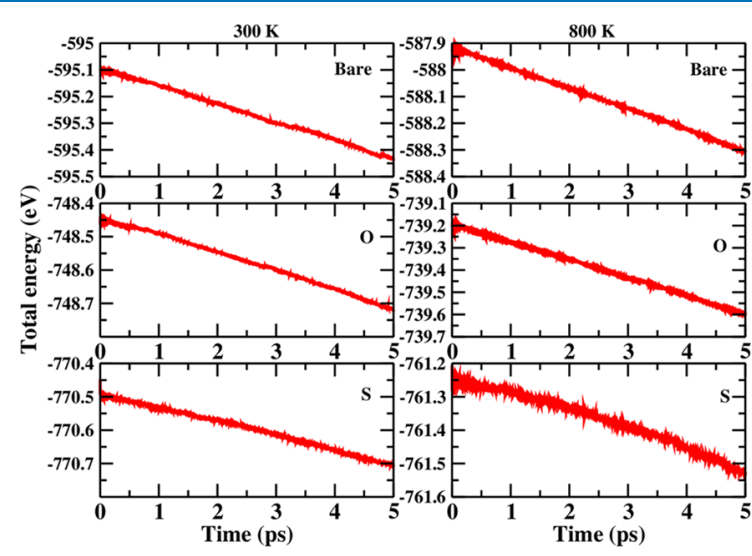

Figure 8. Total energy fluctuations of bare, S-, and O-A-GaNNRs at $300 \mathrm{~K}$ (left) and $800 \mathrm{~K}$ (right) as a function of simulated time.

reveal that the total energy fluctuations for the GaNNRs at 300 and $800 \mathrm{~K}$ are in the $0.2-0.3$ and $0.3-0.4 \mathrm{eV}$ range, respectively. Although atoms vibrate around their equilibrium positions, no structural deformations occur in the geometric structures of bare and S-A-GaNNRs. On the other hand, as the edge $\mathrm{O}$ atoms in the $\mathrm{O}-\mathrm{A}-\mathrm{GaNNR}$ configuration move away from the NR edges, this results in a long range of fluctuations. At high temperatures, the S-A-GaNNR is the most stable configuration among the three NRs studied here, indicating that introducing $S$ during GaNNR fabrication is necessary for improving material stability.

\section{CONCLUSIONS}

In this work, the electronic and magnetic properties of bare AGaNNRs and $\mathrm{O}$ - and $\mathrm{S}-\mathrm{A}-\mathrm{GaNNRs}$ were investigated by means of first-principles calculations both in the absence and under the influence of an external electric field of $0.1-1 \mathrm{~V} / \AA$ strength. Our findings revealed that the band gap of bare AGaNNRs decreased with increasing field strength and vanished at a critical value. Beyond this threshold, the A-GaNNRs exhibited magnetic properties, and the material properties were transformed from semiconducting to metallic nature upon increasing the electric field. A half-metallic material was obtained by further increasing the external electric field. We found that the O-A-GaNNRs were spin-polarized magnetic materials with a finite magnetic moment and existed in both FM and AFM phases. On the other hand, S-A-GaNNRs were nonmagnetic, but their band structure could be transformed from a direct into an indirect band gap. However, S-AGaNNRs exhibited metallic nature under lower electric field strength than that required for their bare counterparts. The formation energy calculations indicated that the passivated NRs were more stable than their bare counterparts. AIMD simulations at 300 and $800 \mathrm{~K}$ further showed that all AGaNNRs studied were stable at room temperature. The S-A-
GaNNR was the most stable configuration at high temperatures among all A-GaNNRs. Therefore, the results reported here showed that A-GaNNRs can be good candidates for a wide range of electronic, optoelectronic, and spintronic device applications. It may not be presently possible to obtain such a large electric field to operate devices; however, it can be suitable for potential applications requiring a very small electric field in the future.

\section{ASSOCIATED CONTENT}

\section{S Supporting Information}

The Supporting Information is available free of charge at https://pubs.acs.org/doi/10.1021/acsomega.9b03841.

A brief statement in non-sentence format listing the contents of the material supplied; band structures of bare 7-A-GaNNRs under the electric field; projected density of states of nonmagnetic, ferromagnetic, and antiferromagnetic O-A-GaNNRs; projected density of states of S-A-GaNNRs; density of states of O-AGaNNRs, S-A-GaNNRs under the electric field; band gap behavior of 7-A-GaNNRs as a function of electric field using the HSE functional (PDF)

\section{AUTHOR INFORMATION}

\section{Corresponding Author}

*E-mail: iman.roqan@kaust.edu.sa.

ORCID ${ }^{\circ}$

Iman S. Roqan: 0000-0001-7442-4330

Notes

The authors declare no competing financial interest.

\section{ACKNOWLEDGMENTS}

N.A. and I.S.R. gratefully acknowledge the supercomputing facility at King Abdullah University of Science and Technology (KAUST) for providing the computational resources to carry out this research work. This work was funded by the base fund BAS/1/1319-01-01.

\section{REFERENCES}

(1) Novoselov, K.; Geim, A.; Morozov, S.; Jiang, D.; Zhang, Y.; Dubonos, S.; Grigorieva, I.; Firsov, A. Electric Field Effect in Atomically Thin Carbon Films. Science 2004, 306, 666-669.

(2) Castro Neto, A. H.; Guinea, F.; Peres, N. M. R.; Novoselov, K. S.; Geim, A. K. The Electronic Properties of Graphene. Rev. Mod. Phys. 2009, 81, 109-162.

(3) Geim, A. K.; Novoselov, K. S. The Rise of Graphene. Nat. Mater. 2007, 6, 183-191.

(4) Katsnelson, M. I. Graphene: Carbon in Two Dimensions. Mater. Today 2007, 10, 20-27.

(5) Vogt, P.; De Padova, P.; Quaresima, C.; Avila, J.; Frantzeskakis, E.; Asensio, M. C.; Resta, A.; Ealet, B.; Le Lay, G. Silicene: Compelling Experimental Evidence for Graphenelike Two-Dimensional Silicon. Phys. Rev. Lett. 2012, 108, No. 155501.

(6) Wang, Q. H.; Kalantar-Zadeh, K.; Kis, A.; Coleman, J. N.; Strano, M. S. Electronics and Optoelectronics of Two-Dimensional Transition Metal Dichalcogenides. Nat. Nanotechnol. 2012, 7, 699712.

(7) Mannix, A. J.; Zhou, X.-F.; Kiraly, B.; Wood, J. D.; Alducin, D.; Myers, B. D.; Liu, X.; Fisher, B. L.; Santiago, U.; Guest, J. R.; et al. Synthesis of Borophenes: Anisotropic, Two-Dimensional Boron Polymorphs. Science 2015, 350, 1513-1516.

(8) Liu, H.; Neal, A. T.; Zhu, Z.; Luo, Z.; Xu, X.; Tománek, D.; Ye, P. D. Phosphorene: An Unexplored 2D Semiconductor with a High Hole Mobility. ACS Nano 2014, 8, 4033-4041. 
(9) Naguib, M.; Mashtalir, O.; Carle, J.; Presser, V.; Lu, J.; Hultman, L.; Gogotsi, Y.; Barsoum, M. W. Two-Dimensional Transition Metal Carbides. ACS Nano 2012, 6, 1322-1331.

(10) Ci, L.; Song, L.; Jin, C.; Jariwala, D.; Wu, D.; Li, Y.; Srivastava, A.; Wang, Z. F.; Storr, K.; Balicas, L.; et al. Atomic Layers of Hybridized Boron Nitride and Graphene Domains. Nat. Mater. 2010, 9, 430-435.

(11) Sun, H.; Shakfa, M. K.; Muhammed, M. M.; Janjua, B.; Li, K.H.; Lin, R.; Ng, T. K.; Roqan, I. S.; Ooi, B. S.; Li, X. SurfacePassivated AlGaN Nanowires for Enhanced Luminescence of Ultraviolet Light Emitting Diodes. ACS Photonics 2018, 5, 964-970.

(12) Flemban, T. H.; Sequeira, M. C.; Zhang, Z.; Venkatesh, S.; Alves, E.; Lorenz, K.; Roqan, I. S. A Identifying the influence of the intrinsic defects in Gd-doped $\mathrm{ZnO}$ thin-films. J. Appl. Phys. 2016, 119, No. 065301.

(13) Wu, F.; Sun, H.; Ajia, I. A.; Roqan, I. S.; Zhang, D.; Dai, J.; Chen, C.; Chuan, Z.; Feng, LiX. Significant internal quantum efficiency enhancement of $\mathrm{GaN} / \mathrm{AlGaN}$ multiple quantum wells emitting at $\sim 350 \mathrm{~nm}$ via step quantum well structure design. J. Phys. D.: Appl. Phys. 2017, 50, No. 245101.

(14) Dolui, K.; Pemmaraju, C. D.; Sanvito, S. Electric Field Effects on Armchair $\mathrm{MoS}_{2}$ Nanoribbons. ACS Nano 2012, 6, 4823-4834.

(15) Xia, Y.; Yang, P.; Sun, Y.; Wu, Y.; Mayers, B.; Gates, B.; Yin, Y.; Kim, F.; Yan, H. One-Dimensional Nanostructures: Synthesis, Characterization, and Applications. Adv. Mater. 2003, 15, 353-389.

(16) Wang, X.; Li, Y. Solution-Based Synthetic Strategies for 1-D Nanostructures. Inorg. Chem. 2006, 45, 7522-7534.

(17) Son, Y.-W.; Cohen, M. L.; Louie, S. G. Energy Gaps in Graphene Nanoribbons. Phys. Rev. Lett. 2006, 97, No. 216803.

(18) Alaal, N.; Loganathan, V.; Medhekar, N.; Shukla, A. First Principles Many-Body Calculations of Electronic Structure and Optical Properties of SiC Nanoribbons. J. Phys. D.: Appl. Phys. 2016, 49, No. 105306

(19) Kou, L.; Tang, C.; Zhang, Y.; Heine, T.; Chen, C.; Frauenheim, T. Tuning Magnetism and Electronic Phase Transitions by Strain and Electric Field in Zigzag $\mathrm{MoS}_{2}$ Nanoribbons. J. Phys. Chem. Lett. 2012, 3, 2934-2941.

(20) Prezzi, D.; Varsano, D.; Ruini, A.; Marini, A.; Molinari, E. Optical Properties of Graphene Nanoribbons: The Role of ManyBody Effects. Phys. Rev. B 2008, 77, No. 041404.

(21) Alaal, N.; Loganathan, V.; Medhekar, N.; Shukla, A. From HalfMetal to Semiconductor: Electron-Correlation Effects in Zigzag SiC Nanoribbons From First Principles. Phys. Rev. Appl. 2017, 7, No. 064009 .

(22) Yagmurcukardes, M.; Peeters, F. M.; Senger, R. T.; Sahin, H. Nanoribbons: From Fundamentals to State-of-the-Art Applications. Appl. Phys. Rev. 2016, 3, No. 041302.

(23) Ren, Y.; Cheng, F.; Zhang, Z. H.; Zhou, G. Half Metal Phase in the Zigzag Phosphorene Nanoribbon. Sci. Rep. 2018, 8, No. 2932.

(24) Alfaraj, N.; Mitra, S.; Wu, F.; Ajia, I. A.; Janjua, B.; Prabaswara, A.; Aljefri, R. A.; Sun, H.; Ng, T. K.; Ooi, B. S.; Roqan, I. S.; Li, X. Photoinduced entropy of InGaN/GaN pin double heterostructure nanowires. Appl. Phys. Lett. 2017, 110, No. 161110.

(25) Carignano, M. A.; Aravindh, S. A.; Roqan, I. S.; Even, J.; Katan, C. Critical Fluctuations and Anharmonicity in Lead Iodide Perovskites from Molecular Dynamics Supercell Simulations. J. Phys. Chem. C 2017, 121, 20729-20738.

(26) Ajia, I. A.; Edwards, P. R.; Pak, Y.; Belekov, E.; Roldan, M. A.; Wei, N.; Liu, Z.; Martin, R. W.; Roqan, I. S. Generated Carrier Dynamics in V-Pit-Enhanced InGaN/GaN Light-Emitting Diode. ACS Photonics 2018, 5, 820-826.

(27) Muhammed, M. M.; Alwadai, N.; Lopatin, S.; Kuramata, A.; Roqan, I. S. High-Efficiency InGaN/GaN Quantum Well-Based Vertical Light-Emitting Diodes Fabricated on $\beta-\mathrm{Ga}_{2} \mathrm{O}_{3}$ Substrate. ACS Appl. Mater. Interfaces 2017, 9, 34057-34063.

(28) Ajia, I. A.; Yamashita, Y.; Lorenz, K.; Muhammed, M. M.; Spasevski, L.; Almalawi, D.; Xu, J.; Iizuka, K.; Morishima, Y.; Anjum, D. H.; et al. GaN/AlGaN Multiple Quantum Wells Grown on Transparent and Conductive (-201)-Oriented $\beta-\mathrm{Ga}_{2} \mathrm{O}_{3}$ Substrate for
UV Vertical Light Emitting Devices. Appl. Phys. Lett. 2018, 113, No. 082102

(29) Alaal, N.; Roqan, I. S. Tuning the Electronic Properties of Hexagonal Two-Dimensional GaN Monolayers via Doping for Enhanced Optoelectronic Applications. ACS Appl. Nano Mater. 2019, 2, 202-213.

(30) Şahin, H.; Cahangirov, S.; Topsakal, M.; Bekaroglu, E.; Akturk, E.; Senger, R. T.; Ciraci, S. Monolayer Honeycomb Structures of Group-IV Elements and III-V Binary Compounds: First-Principles Calculations. Phys. Rev. B 2009, 80, No. 155453.

(31) Al Balushi, Z. Y.; Wang, K.; Ghosh, R. K.; Vilá, R. A.; Eichfeld, S. M.; Caldwell, J. D.; Qin, X.; Lin, Y.-C.; DeSario, P. A.; Stone, G.; et al. Two-Dimensional Gallium Nitride Realized via Graphene Encapsulation. Nat. Mater. 2016, 15, 1166-1171.

(32) Yang, L.; Zhang, X.; Huang, R.; Zhang, G.; An, X. Synthesis of Single Crystalline GaN Nanoribbons on Sapphire (0001) Substrates. Solid State Commun. 2004, 130, 769-772.

(33) Li, H.; Dai, J.; Li, J.; Zhang, S.; Zhou, J.; Zhang, L.; Chu, W.; Chen, D.; Zhao, H.; Yang, J.; et al. Electronic Structures and Magnetic Properties of GaN Sheets and Nanoribbons. J. Phys. Chem. C 2010, 114, 11390-11394.

(34) Li, S.; Xing, H.; Xie, R.; Zeng, Y.; Huang, Y.; Lu, A.; Chen, X. Electronic and Magnetic Properties of Zigzag GaN Nanoribbons with Hydrogenation and Fluorination. Phys. E 2018, 97, 144-150.

(35) Zheng, F.-L.; Zhang, Y.; Zhang, J.-M.; Xu, K.-W. Structural, Electronic, and Magnetic Properties of C-Doped GaN Nanoribbon. J. Appl. Phys. 2011, 109, No. 104313.

(36) Kresse, G.; Furthmüller, J. Efficient Iterative Schemes for Ab Initio Total-Energy Calculations Using a Plane-Wave Basis Set. Phys. Rev. B 1996, 54, 11169-11186.

(37) Kresse, G.; Furthmüller, J. Efficiency of $\backslash \operatorname{emph}\{a b$ Initio $\}$ Total Energy Calculations for Metals and Semiconductors Using a PlaneWave Basis Set. Comput. Mater. Sci. 1996, 6, 15-50.

(38) Perdew, J. P.; Burke, K.; Ernzerhof, M. Generalized Gradient Approximation Made Simple. Phys. Rev. Lett. 1996, 77, 3865-3868.

(39) Momma, K.; Izumi, F. VESTA: A Three-Dimensional Visualization System for Electronic and Structural Analysis. J Appl Crystallogr 2008, 41, 653-658.

(40) Hoover, W. G. Canonical Dynamics: Equilibrium Phase-Space Distributions. Phys. Rev. A 1985, 31, 1695-1697.

(41) Özçelik, V. O.; Azadani, J. G.; Yang, C.; Koester, S. J.; Low, T. Band Alignment of Two-Dimensional Semiconductors for Designing Heterostructures with Momentum Space Matching. Phys. Rev. B 2016, 94, No. 035125

(42) Little, E. J.; Jones, M. M. A Complete Table of Electronegativities. J. Chem. Educ. 1960, 37, No. 231.

(43) Zhang, Z.; Guo, W. Energy-Gap Modulation of BN Ribbons by Transverse Electric Fields: First-Principles Calculations. Phys. Rev. B 2008, 77, No. 075403.

(44) O’Keeffe, J.; Wei, C.; Cho, K. Bandstructure Modulation for Carbon Nanotubes in a Uniform Electric Field. Appl. Phys. Lett. 2002, 80, 676-678.

(45) Loh, S. M.; Huang, Y.-H.; Lin, K.-M.; Su, W. S.; Wu, B. R.; Leung, T. C. Quantum Confinement Effect in Armchair Graphene Nanoribbons: Effect of Strain on Band Gap Modulation Studied Using First-Principles Calculations. Phys. Rev. B 2014, 90, No. 035450.

(46) Bantounas, I.; Singaravelu, V.; Roqan, I. S.; Schwingenschlögl, U. Structural and Magnetic Properties of Gd-Doped ZnO. J. Mater. Chem. C 2014, 2, 10331-10336.

(47) Zhang, Z.; Schwingenschlögl, U.; Roqan, I. S. Possible mechanism for $\mathrm{d}^{0}$ ferromagnetism mediated by intrinsic defects. RSC Adv. 2014, 4, 50759-50764.

(48) Aravindh, S. D. A.; Schwingenschlögl, U.; Roqan, I. S. Defect induced $\mathrm{d}^{0}$ ferromagnetism in a $\mathrm{ZnO}$ grain boundary. J. Chem. Phys. 2015, 143, No. 224703.

(49) Son, Y.-W.; Cohen, M. L.; Louie, S. G. Half-Metallic Graphene Nanoribbons. Nature 2006, 444, 347-349. 
(50) Barone, V.; Peralta, J. E. Magnetic Boron Nitride Nanoribbons with Tunable Electronic Properties. Nano Lett. 2008, 8, 2210-2214.

(51) Dutta, S.; Manna, A. K.; Pati, S. K. Intrinsic Half-Metallicity in Modified Graphene Nanoribbons. Phys. Rev. Lett. 2009, 102, No. 096601.

(52) Tang, Q.; Zhou, Z.; Chen, Z. Graphene-Related Nanomaterials: Tuning Properties by Functionalization. Nanoscale 2013, 5, No. 4541.

(53) Lee, G.; Cho, K. Electronic Structures of Zigzag Graphene Nanoribbons with Edge Hydrogenation and Oxidation. Phys. Rev. B 2009, 79, No. 165440.

(54) Lopez-Bezanilla, A.; Huang, J.; Kent, P. R. C.; Sumpter, B. G. Tuning from Half-Metallic to Semiconducting Behavior in $\mathrm{SiC}$ Nanoribbons. J. Phys. Chem. C 2013, 117, 15447-15455.

(55) Wan, L. F.; Cho, E. S.; Marangoni, T.; Shea, P.; Kang, S.; Rogers, C.; Zaia, E.; Cloke, R. R.; Wood, B. C.; Fischer, F. R.; et al. Edge-Functionalized Graphene Nanoribbon Encapsulation To Enhance Stability and Control Kinetics of Hydrogen Storage Materials. Chem. Mater. 2019, 31, 2960-2970.

(56) Chamberlain, T. W.; Biskupek, J.; Rance, G. A.; Chuvilin, A.; Alexander, T. J.; Bichoutskaia, E.; Kaiser, U.; Khlobystov, A. N. Size, Structure, and Helical Twist of Graphene Nanoribbons Controlled by Confinement in Carbon Nanotubes. ACS Nano 2012, 6, 3943-3953.

(57) Caridad, J. M.; Calogero, G.; Pedrinazzi, P.; Santos, J. E.; Impellizzeri, A.; Gunst, T.; Booth, T. J.; Sordan, R.; Bøggild, P.; Brandbyge, M. A Graphene-Edge Ferroelectric Molecular Switch. Nano Lett. 2018, 18, 4675-4683.

(58) Jin, J.; Wang, Z.; Dai, X.; Xiao, J.; Long, M.; Chen, T. The Electronic and Transport Properties of the Phosphorene Nanoribbons. Mater. Res. Express 2019, 6, No. 096317.

(59) Alaal, N.; Medhekar, N.; Shukla, A. Tunable Electronic Properties of Partially Edge-Hydrogenated Armchair Boron-Nitrogen-Carbon Nanoribbons. Phys. Chem. Chem. Phys. 2018, 20, 10345-10358.

(60) Paier, J.; Marsman, M.; Hummer, K.; Kresse, G.; Gerber, I. C.; Ángyán, J. G. Screened Hybrid Density Functionals Applied to Solids. J. Chem. Phys. 2006, 124, No. 154709.

(61) Hod, O.; Barone, V.; Peralta, J. E.; Scuseria, G. E. Enhanced Half-Metallicity in Edge-Oxidized Zigzag Graphene Nanoribbons. Nano Lett. 2007, 7, 2295-2299.

(62) Jain, A.; Ong, S. P.; Hautier, G.; Chen, W.; Richards, W. D.; Dacek, S.; Cholia, S.; Gunter, D.; Skinner, D.; Ceder, G.; et al. Commentary: The Materials Project: A Materials Genome Approach to Accelerating Materials Innovation. APL Mater. 2013, 1, No. 011002. 